\title{
Long-noncoding RNA NBR2 via targeting miR-19a-3p regulates pancreatic $\beta$ cell function and provides novel biomarkers for type 2 diabetes mellitus
}

\section{Type}

Research paper

\section{Keywords}

Diagnosis, Proliferation, NBR2, MiR-19a-3p, Type 2 diabetes mellitus, Pancreatic $\beta$ cell, Insulin secretion

\begin{abstract}
Introduction

Type 2 diabetes mellitus (T2DM) is a major kind of diabetes mellitus. This study aimed to analyze the regulatory effect of long noncoding RNA NBR2 on pancreatic $\square$ cell function and the related mechanisms, and to analyze the clinical significance of abnormal NBR2 expression in patients with T2DM.
\end{abstract}

\section{Material and methods}

The expression levels of NBR2 and microRNA-19a-3p (miR-19a-3p) were measured using quantitative Real-Time PCR. The glucose-stimulated insulin secretion was measured using ELISA kit, and cell proliferation was examined using Cell Counting Kit-8 (CCK-8) assay. A dual-luciferase reporter assay was used to confirm the relationship between NBR2 and miR-19a-3p. The diagnostic values of NBR2, miR-19a-3p and NBR2 combined with miR-19a-3p were assessed by receiver operating characteristic (ROC) curves.

\section{Results}

The insulin secretion and proliferation of INS-1 cells were inhibited by NBR2 overexpression, and were promoted by NBR2 knockdown. MiR-19a-3p, which was inhibited by NBR2 overexpression, directly mediated the regulatory effects of NBR2 on INS-1 cell function. Increased serum NBR2 level and decreased serum miR-19a-3p level were found in T2DM patients, and a negative correlation was found between NBR2 and miR-19a-3p. The fasting plasma glucose of T2DM patients was positively correlated with serum NBR2 and negatively correlated with serum miR-19a-3p. Both serum NBR2 and miR-19a-3p had certain diagnostic accuracy, whereas, the combined detection of the serum NBR2 and miR-19a-3p showed more considerable diagnostic accuracy.

\section{Conclusions}

Our findings indicated that NBR2/miR-19a-3p axis regulates pancreatic $\beta$ cell function, while may be novel biomarkers for the diagnosis of T2DM. 
Long-noncoding RNA NBR2 via targeting miR-19a-3p regulates pancreatic $\beta$ cell function and provides novel biomarkers for type 2 diabetes mellitus

Short title: Role of the NBR2/miR-19a-3p axis in T2DM

Lei Wan ${ }^{1}$, Xinying Song ${ }^{2 *}$, Baoqing $\mathrm{Zhu}^{2}$

${ }^{1}$ Endocrinology Department, Affiliated Hospital of Weifang Medical University, Weifang 261021, Shandong, China.

${ }^{2}$ Endocrinology Department, Zhucheng People's Hospital, Zhucheng 262200, Shandong, China.

*The author is the corresponding author.

\section{*Corresponding Author}

Xinying Song, Endocrinology Department, Zhucheng People's Hospital, No. 59, South Ring Road, Zhucheng, 262200, Shandong, China. E-mail: songxy_zcph@163.com; Tel/Fax: 0536-6026176. 


\begin{abstract}
Introduction: Type 2 diabetes mellitus (T2DM) is a major kind of diabetes mellitus. This study aimed to analyze the regulatory effect of long noncoding RNA NBR2 on pancreatic $\beta$ cell function and the related mechanisms, and to analyze the clinical significance of abnormal NBR2 expression in patients with T2DM.
\end{abstract}

Material and methods: The expression levels of NBR2 and microRNA-19a-3p (miR-19a-3p) were measured using quantitative Real-Time PCR. The glucose-stimulated insulin secretion was measured using ELISA kit, and cell proliferation was examined using Cell Counting Kit-8 (CCK-8) assay. A dualluciferase reporter assay was used to confirm the relationship between NBR2 and miR-19a-3p. The diagnostic values of NBR2, miR-19a-3p and NBR2 combined with miR-19a-3p were assessed by receiver operating characteristic (ROC) curves.

Results: The insulin secretion and proliferation of INS-1 cells were inhibited by NBR2 overexpression, and were promoted by NBR2 knockdown. MiR-19a-3p, which was inhibited by NBR2 overexpression, directly mediated the regulatory effects of NBR2 on INS-1 cell function. Increased serum NBR2 level and decreased serum miR-19a-3p level were found in T2DM patients, and a negative correlation was found between NBR2 and miR-19a-3p. The fasting plasma glucose of T2DM patients was positively correlated with serum NBR2 and negatively correlated with serum miR-19a-3p. Both serum NBR2 and miR-19a-3p had certain diagnostic accuracy, whereas, the combined detection of the serum NBR2 and miR-19a-3p showed more considerable diagnostic accuracy.

Conclusions: Our findings indicated that NBR2/miR-19a-3p axis regulates pancreatic $\beta$ cell function, while may be novel biomarkers for the diagnosis of T2DM.

Keywords: NBR2; MiR-19a-3p; Type 2 diabetes mellitus; Pancreatic $\beta$ cell; Proliferation; Insulin secretion; Diagnosis 


\section{Introduction}

Type 2 diabetes mellitus (T2DM) is characterized by insulin resistance or insufficient insulin secretion, leading to hyperglycemia, which accounts for approximately $90 \%$ of all types of diabetes mellitus $(1,2)$. The global prevalence of T2DM continues to rise, with corresponding increases in morbidity and mortality, placing an increasing burden on the health care system (3). The pancreatic $\beta$ cells play key role in glucose homeostasis by secreting insulin, the only hormone capable of lowering the blood glucose concentration (4).In addition, the abnormal function of pancreatic $\beta$ cells was mainly manifested as insensitivity to the elevation of blood glucose and the weakened ability of insulin secretion (5). Thus, the $\beta$ cell function was thought to play a major role in the pathogenesis of T2DM (6). Therefore, exploring ways to improve pancreatic $\beta$-cells may help improve T2DM.

Long noncoding RNAs (LncRNAs) are over 200 nucleotides in length and can regulate gene expression at epigenetic, transcriptional, and posttranscriptional levels (7). LncRNAs have been increasingly recognized to regulate pancreatic $\beta$ cell development and insulin secretion. For example, Feng et al. found that LncRNA DANCR plays a crucial role in the regulation of the cell proliferation and insulin production of INS-1 cells (8). The overexpression of LINC-P21 inhibited insulin secretion in response to glucose stimulation and the proliferation of INS-1 cells (9). In addition, it is noteworthy that in a study of LncRNAs associated with insulin resistance in T2DM, NBR2 was found to significantly increase in T2DM patients (10), however, the role of NBR2 in the pathological mechanism of T2DM has not been deeply studied in this study. Besides, in the previous study, the biological effects and clinical values of NBR2 on $\beta$ cells in T2DM have not been studied.

MicroRNAs (miRNAs) are small 22-25 nucleotides long non-coding RNAs and regulate target gene expression by degrading the corresponding mRNA and/or suppressing their translation (11). A study summarized the Previous studies have found that miRNAs are related to the regulation of pancreatic $\beta$ cell function. For example, microRNA-96 overexpression could enhance proliferation and suppress apoptosis of pancreatic $\beta$ cell (12). Song et al. found that microRNA-26a overexpression might prevented the $\beta$ cell dysfunction (13). And microRNA-125b-5p upregulation enhanced the insulin sensitivity and elevate the pancreatic $\beta$ cell proliferation (14). Moreover, microRNA-19a-3p (miR-19a-3p) was demonstrated to be bound by NBR2, mediating the biological function of NBR2 in acute liver failure (ALF) (15). Furthermore, miR-19a-3p was found to promote pancreatic $\beta$-cell proliferation and insulin secretion (16). Therefore, we speculated that NBR2 may also have a relationship with the function of pancreatic $\beta$ cell.

Thus, this study aimed to analyze the regulatory effect of NBR2 on pancreatic $\beta$ cell proliferation and insulin secretion, and the related mechanisms, and to evaluate the clinical significance of abnormal expression of NBR2 in patients with T2DM. It is hoped to provide new biomarkers and molecular targets 
for the diagnosis and treatment of T2DM on the basis of further understanding of the pathological mechanism of T2DM.

\section{Materials and methods}

\section{Cell culture and transfection}

The pancreatic $\beta$ cell line INS-1 was purchased from the Cell Bank of Type Culture Collection of Chinese Academy of Sciences (Shanghai, China). Then they were cultured in Dulbecco's modified Eagle's medium (DMEM, Invitrogen, Thermo Fisher Scientific, CA, USA) supplemented with 10\% fetal bovine serum (FBS, Gibco, CA, USA), and maintained in a humidified incubator with $5 \% \mathrm{CO}_{2}$ at $37^{\circ} \mathrm{C}$. The pcDNA3.1-NBR2, NBR2 small interfering RNA (NBR2 siRNA), its negative control (NC siRNA), miR-19a-3p mimic and the mimic negative control (mimic NC) were synthesized from GenePharma (Shanghai, China). The above vectors were transfected into INS-1 cells using Lipofectamine 3000 (Invitrogen, Carlsbad, CA, USA) according to the manufacturer's protocols, and were used for the subsequent experimental analyses after $48 \mathrm{~h}$ of transfection.

Patients and serum sample collection

A total of 106 T2DM patients admitted to Affiliated Hospital of Weifang Medical University from 2017 to 2019 were recruited as study subjects. In addition, 62 healthy volunteers who received physical examination and had normal glucose tolerance were recruited as healthy controls. Venous blood was collected from each participant and centrifuged for the extraction of serum, which was stored at $-80^{\circ} \mathrm{C}$ for further use. The T2DM was diagnosed based on the fast plasma glucose (FPG) test (blood samples were collected after $12 \mathrm{~h}$ of fasting) and OGTT examination. The subjects who have FPG $\geq 7.0 \mathrm{mM}$ $(126 \mathrm{mg} / \mathrm{dL})$ and $/$ or $2 \mathrm{~h}$ plasma glucose value $(2 \mathrm{~h} \mathrm{PG}) \geq 11.1 \mathrm{mM}(200 \mathrm{mg} / \mathrm{dL})$ from the OGTT test were diagnosed as T2DM patients (17). The demographic and general clinical data of the subjects were recorded and compared and showed in Table 1, indicating that T2DM patients and healthy controls were matched in terms of age, gender and body mass index (BMI), and the general clinical data included total cholesterol (TC), triglyceride (TG), high density lipoproteins (HDL), low density lipoproteins (LDL), glycosylated hemoglobin (HbAlc), and oral glucose tolerance test (OGTT) at $0 \mathrm{~h}$ and $2 \mathrm{~h}$. This study was approved by the Ethics Committee of Affiliated Hospital of Weifang Medical University and each participant provided a written informed consent.

\section{$R N A$ extraction and quantitative real-time PCR $(q R T-P C R)$}

The TRIzol reagent (Invitrogen, Carlsbad, CA, USA) was used to extract total RNA from INS-1 cells and serum. The purity and concentration of the RNA were evaluated by a NanoDrop 2000 (Thermo 
Fisher Scientific, Inc.). First-stranded cDNA was synthesized using a PrimeScript RT reagent kit (TaKaRa, Japan).

The levels of NBR2 and miR-19a-3p were measured using qRT-PCR, which was carried out using a SYBR Green I Master Mix kit (Invitrogen, Thermo Fisher Scientific, Inc.) and a 7300 Real-Time PCR System (Applied Biosystems; Thermo Fisher Scientific, Inc.). All the protocols were performed following the manufacturer's instructions. The NBR2 and miR-19a-3p expression were normalized to GAPDH and U6, respectively. The primer sequences used for qRT-PCR were presented in Table 2. Relative expression levels were calculated using $2^{-\Delta \Delta \mathrm{Ct}}$ method.

\section{Glucose-stimulated insulin secretion assay}

After cell transfection, the INS-1 cells were seeded into 96-well plates and further cultured for $24 \mathrm{~h}$. Then the cells were treated with a concentration of $3.3 \mathrm{mM}$ and $16.7 \mathrm{mM}$ glucose for $1 \mathrm{~h}$ to stimulate insulin secretion. And secretion of insulin was detected using ELISA kit.

\section{Cell proliferation assay}

After cell transfection, the cell proliferation was examined using the Cell Counting Kit-8 (CCK-8). The stable transfected INS-1 cells were seeded into 96 -well plates at a density of $3 \times 10^{3}$ cells/well, and then cultured in a humidified incubator at $37^{\circ} \mathrm{C}$. The CCK-8 reagent was added in the cells after incubated for 24,48 and $72 \mathrm{~h}$, and was further incubated for $2 \mathrm{~h}$. The cell proliferation was measured by reading the absorbance at a wavelength of $450 \mathrm{~nm}$ using a micro-plate analyzer (Bio-Rad Laboratories, Inc.).

\section{Dual-luciferase reporter assay}

The putative binding site of miR-19a-3p was found at the sequence of NBR2, and a dual-luciferase reporter assay was conducted. The wild-type (WT) 3'-UTR containing the binding site of miR-19a-3p or mutant-type (MUT) 3'-UTR were integrated into the luciferase reporter vector (NBR2-WT and NBR2MUT). Then the NBR2-WT and NBR2-MUT were separately co-transfected into INS-1 cells with miR19a-3p mimic or mimic NC using Lipofectamine 3000 reagent (Invitrogen, Carlsbad, CA, USA). After 48h transfection, relative luciferase activity was measured using a Dual-Luciferase Reporter Assay System (Promega). All the procedures followed the manufacturer's instructions.

\section{Statistical analysis}

All the statistical analyses were carried out by SPSS 21.0 software (SPSS, Inc., Chicago, USA) and GraphPad Prism 7.0 software (Inc., Chicago, USA). All data were presented as mean \pm standard deviation (SD). Differences between groups were analyzed using Student's t test, Chi-square test or one-way ANOVA followed by Tukey's test. The correlation between indicators in T2DM patients was assessed 
using Pearson correlation coefficient. The receiver operating characteristic (ROC) curves were plotted to assess the diagnostic value of NBR2, miR-19a-3p and NBR2 + miR-19a-3p. A $P<0.05$ indicated statistically significant.

\section{Results}

\section{Baseline characteristics of the study subjects}

The baseline characteristics of the patients with T2DM $(n=106)$ and healthy subjects $(n=62)$ were included in Table 1. No differences were observed between T2DM patients and healthy subjects in gender, age, BMI, TC, TG, HDL and LDL (all $P>0.05$ ), while the T2DM patients had significant elevated levels of $\mathrm{HbA1} \mathrm{c}$ and high OGTT (at $0 \mathrm{~h}$ and $2 \mathrm{~h}$ ) results compared with the healthy subjects (all $P<0.001)$.

\section{Effects of NBR2 on pancreatic $\beta$ cell insulin secretion and proliferation}

The expression level of NBR2 was measured using qRT-PCR. As shown in Figure 1A, the expression of NBR2 in the INS-1 cells was increased in cells transfected with pcDNA3.1-NBR2, while was decreased in cells transfected with NBR2 siRNA (all $P<0.001$ ). The insulin secretion in response to glucose stimulation was significantly decreased in NBR2-overexpressed cells, while was significantly increased in NBR2-silenced cells compared with the secretion of insulin in untransfected cells (Figure 1B, all $P<0.05)$. Then we evaluated the proliferation of INS-1 cells. From the Figure 1C, we found that the proliferation was inhibited by the NBR2 overexpression, and was promoted by the NBR2 knockdown (all $P<0.05$ )

MiR-19a-3p directly mediates the regulatory effects of NBR2 on pancreatic $\beta$ cell function

To confirm the interaction between NBR2 and miR-19a-3p, a luciferase reporter assay was performed. The binding sequence between NBR2 and miR-19a-3p was shown in Figure 2A. As presented in Figure 2B, the miR-19a-3p expression was promoted by the miR-19a-3p mimic compared with that in untransfected cells $(P<0.001)$. In INC-1 cells, the relative luciferase activity in NBR2-WT group was inhibited by miR-19a-3p overexpression $(P<0.05)$, whereas no changes were observed in luciferase activity in NBR2-MUT group (Figure 2C), suggesting the direct binding of miR-19a-3p to NBR2. As shown in Figure 2D, the NBR2 overexpression could inhibit, and NBR2 knockdown could promote the expression of miR-19a-3p in INS-1 cells (all $P<0.001$ ). Then we co-transfected regulatory vectors of NBR2 and miR-19a-3p expression levels in INS-1 cells, and found that miR-19a-3p could significantly reverse the inhibitory effect of NBR2 overexpression on the miR-19a-3p expression (Figure 2E, all $P<$ 0.001). From the Figure $2 \mathbf{F}$ and $\mathbf{G}$, the repressed insulin secretion and cell proliferation caused by NBR2 overexpression were reversed by the overexpression of miR-19a-3p (all $P<0.05$ ). 
Expression of the NBR2/miR-19a-3p axis in patients with T2DM

We detected the expression levels of NBR2 and miR-19a-3p in the serum of healthy volunteers and T2DM patients. As shown in Figure 3A, the expression level of serum NBR2 was markedly upregulated in T2DM patients compared with that in healthy volunteers $(P<0.001)$. And as shown in Figure 3B, the expression level of serum miR-19a-3p was markedly downregulated in T2DM patients compared with that in healthy volunteers $(P<0.001)$. From the Figure 3C, a negative correlation was found between serum miR-19a-3p level and serum NBR2 level $(\mathrm{r}=-0.858, P<0.001)$.

\section{Correlation of the NBR2/miR-19a-3p axis with FPG in T2DM patients}

The correlation of serum NBR2 level and serum miR-19a-3p level with FPG were analyzed in T2DM patients. We found that FPG was positively correlated with the serum NBR2 level (Figure 4A, $r=0.725$, $P<0.001$ ), while was negatively correlated with the serum miR-19a-3p level (Figure 4B, $\mathrm{r}=-0.685, P$ $<0.001)$.

Diagnostic performance of the NBR2/miR-19a-3p axis in patients with T2DM

The ROC curve for the NBR2 level was shown in Figure 5A, and the area under the curve (AUC) was 0.924 with the sensitivity and specificity of $79.25 \%$ and $91.94 \%$, respectively. The AUC for miR-19a-3p level was 0.902 with the sensitivity and specificity of $79.25 \%$ and $87.10 \%$, respectively (Figure 5B). The ROC curve for the NBR2 + miR-19a-3p levels was shown in Figure 5C, and the AUC was 0.945 with the sensitivity and specificity of $86.79 \%$ and $87.10 \%$, respectively. The above results indicated that both serum NBR2 and miR-19a-3p had certain diagnostic accuracy, however, the combined detection of the serum NBR2 and miR-19a-3p revealed more considerable diagnostic accuracy in distinguishing T2DM patients from healthy subjects.

\section{Discussion}

During the pathogenesis of T2DM disease which is a comprehensive metabolic disease, abnormal function of pancreatic $\beta$ cells reduced function of secreting insulin. Consequently, it is necessary to search for the regulatory molecular targets of pancreatic $\beta$ cell function. LncRNAs have been stated to regulate pancreatic $\beta$ cell function. For instance, a study by Wang et al. revealed that lncEif4g was a functional regulator of INS-1E $\beta$ cells in cell viability, insulin production (18). LncRNA-3134 was found to regulate the proliferation and insulin secretion of pancreatic $\beta$ cell (19). However, the role of NBR2 in pancreatic $\beta$ cells remains unclear. In our study, the results indicated that NBR2 overexpression could suppress the insulin secretion and proliferation, NBR2 knockdown promoted the insulin secretion and proliferation. Of note, it has also been reported that NBR2 has the regulatory effects on cell biological function of different cell types. For instance, NBR2 overexpression inhibited the viability and migration of non- 
small cell lung cancer cells (20). A study by Bai et al. revealed that NBR2 could inhibit the proliferation of colorectal cancer cells (21). Therefore, we considered that NBR2 might be a functional molecule in $\beta$ cell function and might be a potential therapeutic target for T2DM by regulating pancreatic $\beta$ cell function.

Growing evidence revealed that the dysregulation of miRNAs could affect the function of pancreatic $\beta$ cell. For example, miR-22 enhanced the viability and inhibited apoptosis of $\beta$ cells (22). The miR$125 \mathrm{~b}-5 \mathrm{p}$ upregulation enhanced insulin sensitivity and promoted pancreatic $\beta$ cell proliferation (14). A study by Wang et al. found that miR-30d upregulation promoted $\beta$ cell proliferation (23). Importantly, Li et al. found that miR-19a-3p promoted pancreatic $\beta$ cell proliferation and insulin secretion (16). And the results of our study also indicated that miR-19a-3p overexpression could promote insulin secretion and proliferation of INS-1 cells. In addition, previous evidence has demonstrated the regulatory effects of miR-19a-3p on cell biological function in different cell types, such as prostate cancer (PCa) cells (24) and osteosarcoma cells (25). Therefore, miR-19a-3p might regulate the $\beta$ cell function. Furthermore, numerous studies have highlighted the mediator role of miRNAs in the mechanisms of LncRNAs biological function $(26,27)$. And miRNAs mediating the effect of LncRNAs on $\beta$ cell biological function has been demonstrated. For instance, Cao et al. found that LINC-P21 could regulate the cell viability and insulin secretion of pancreatic $\beta$ cell, which was mediated by miR-766-3p (9). LncRNA DANCR/miR$33 a-5 p$ axis played important role in regulating the cell growth and insulin production of INS-1 cells (8). In our study, the correlation between NBR2 and miR-19a-3p was found by a luciferase reporter assay. NBR2 overexpression could inhibit the expression of miR-19a-3p in INS-1 cells and the inhibitory effects of NBR2 overexpression on insulin secretion and cell proliferation were reversed by the overexpression of miR-19a-3p. In the ALF, it was also found that miR-19a-3p was bound by NBR2, mediating the biological function of NBR2 (15). Thus, NBR2 might inhibit the proliferation and insulin secretion of INS-1 cells by sponging miR-19a-3p.

After explore the regulatory effect of NBR2/miR-19a-3p on pancreatic $\beta$ cell biological function and the related mechanisms, we evaluated the clinical significance of NBR2/miR-19a-3p axis in patients with T2DM. Increasing studies have documented the important roles of LncRNAs and miRNAs in the progression of T2DM, such as LncRNA NONRATT021972 (28), miR-125a-5p (29), and LINCP21/miR-766-3p (9). Besides, in a study of LncRNAs associated with insulin resistance in T2DM, NBR2 was found to be significantly increased in T2DM patients (10). And our study found the increase in serum NBR2 level and decrease in serum miR-19a-3p level in T2DM patients compared with that in healthy subjects. In addition, positive correlation was found between serum NBR2 level and FPG, and negative correlation was found between serum miR-19a-3p level and FPG in T2DM patients. These findings indicated that NBR2 and miR-19a-3p might be involved in the progression of the T2DM.

Some LncRNAs and miRNAs that aberrantly expressed in disease progression have potentials to serve 
as diagnostic biomarkers for various human diseases, including T2DM. For instance, LncRNA-P21 levels might help to screen PCa from the benign disease (30). The miR-29 family could serve as the diagnosis biomarker of many cancers (31). Besides, LncRNA ENST00000550337.1 (32), miR-181b and miR-126-p (33) have been found to be diagnostic biomarkers for T2DM. Moreover, a review article summarized recent research findings on the roles of lncRNAs in cancers of organs with endocrine functions, including pancreas, ultimately suggesting that lncRNAs may be biomarkers for cancers with organs with endocrine functions (34). In this study, due to the dysregulation of NBR2 and miR-19a-3p levels, we explored the clinical values of them for the diagnosis of T2DM patients using ROC curves. The results revealed that both serum NBR2 and miR-19a-3p had certain diagnostic accuracy, while the detection of serum NBR2 combined with miR-19a-3p revealed more considerable diagnostic accuracy to screen T2DM patients who did not develop any clinical symptoms from healthy subjects. Besides, a study found that NBR2 might be the candidate biomarker for diagnosis of medial temporal lobe epilepsy (35). The diagnostic significance of dysregulation miR-19a-3p has also been identified in other diseases, such as gastric cancer (36) and ovarian cancer (37). Thus, NBR2 and miR-19a-3p might be novel biomarkers for T2DM.

It is known that 19a-3p could regulate pancreatic $\beta$ cell function through inhibiting SOCS3 (16). Besides, a study has indicated that miR-19a negatively regulated autophagy attenuation in hepatocytes by regulating NBR2 and AMPK/PPAR $\alpha$ signaling (15). Therefore, NBR2/miR-19a-3p axis binding the SOCS3 as well as AMPK signaling might be the further mechanism of T2DM pancreatic $\beta$ cells, which will be investigated in further study. Additionally, a limitation of this study was the small sample size and future studies are needed with a large research cohort.

\section{Conclusion}

In conclusion, the overexpression of NBR2 inhibited the insulin secretion and proliferation of pancreatic $\beta$ cell by sponging miR-19a-3p. In addition, the expression of serum NBR2 and miR-19a-3p was abnormal in T2DM patients and might be candidate diagnostic biomarkers to screen T2DM patients who did not develop any clinical symptoms from healthy subjects. Thus, the NBR2/miR-19a-3p axis regulated pancreatic $\beta$ cell function and might provide novel biomarkers for T2DM. The results of this study will help us to further understand the pathogenesis of T2DM, and the development of pharmacological strategies that can regulate the expression of NBR2/miR-19a-3p axis may improve the treatment of T2DM

\section{Ethics approval and consent to participate}

A signed written informed consent was obtained from each patient and the experimental procedures were all in accordance with the guideline of the Ethics Committee of Affiliated Hospital of Weifang Medical 
University.

\section{Consent for publication}

Written informed consent for publication was obtained from each participant.

\section{Availability of data and material}

All data generated or analyzed during this study are included in this published article.

\section{Conflicts of interest}

The authors have declared no conflict interest in this work.

\section{Funding}

None.

\section{Acknowledgements}

Not applicable.

\section{References}

1. Zhou Z, Wang L, Wen Z, Zheng S, Ye X, Liu D, et al. Association Analysis of NLRP3 InflammationRelated Gene Promotor Methylation as Well as Mediating Effects on T2DM and Vascular Complications in a Southern Han Chinese Population. Frontiers in endocrinology. 2018;9:709.

2. Golsheh S, Keshavarzi F. Genetic variants linked to T2DM risk in Kurdish populations. Diabetes, metabolic syndrome and obesity : targets and therapy. 2019;12:431-7.

3. Ying W, Fu W, Lee YS, Olefsky JM. The role of macrophages in obesity-associated islet inflammation and beta-cell abnormalities. Nature reviews Endocrinology. 2020;16(2):81-90.

4. Rorsman P, Ashcroft FM. Pancreatic beta-Cell Electrical Activity and Insulin Secretion: Of Mice and Men. Physiological reviews. 2018;98(1):117-214.

5. Gerber PA, Rutter GA. The Role of Oxidative Stress and Hypoxia in Pancreatic Beta-Cell Dysfunction in Diabetes Mellitus. Antioxidants \& redox signaling. 2017;26(10):501-18.

6. Kitamura T. The role of FOXO1 in beta-cell failure and type 2 diabetes mellitus. Nature reviews Endocrinology. 2013;9(10):615-23.

7. Luo Q, Chen Y. Long noncoding RNAs and Alzheimer's disease. Clinical interventions in aging. 2016;11:867-72. 
8. Feng Y, Qu X, Chen Y, Feng Q, Zhang Y, Hu J, et al. MicroRNA-33a-5p sponges to inhibit pancreatic beta-cell function in gestational diabetes mellitus LncRNA DANCR. Reproductive biology and endocrinology : RB\&E. 2020;18(1):61.

9. Cao Z, Yao F, Lang Y, Feng X. Elevated Circulating LINC-P21 Serves as a Diagnostic Biomarker of Type 2 Diabetes Mellitus and Regulates Pancreatic beta-cell Function by Sponging miR-766-3p to Upregulate NR3C2. Experimental and clinical endocrinology \& diabetes : official journal, German Society of Endocrinology [and] German Diabetes Association. 2020.

10. Sathishkumar C, Prabu P, Mohan V, Balasubramanyam M. Linking a role of lncRNAs (long noncoding RNAs) with insulin resistance, accelerated senescence, and inflammation in patients with type 2 diabetes. Human genomics. 2018;12(1):41.

11. Correia de Sousa M, Gjorgjieva M, Dolicka D, Sobolewski C, Foti M. Deciphering miRNAs' Action through miRNA Editing. International journal of molecular sciences. 2019;20(24).

12. Qi H, Yao L, Liu Q. MicroRNA-96 regulates pancreatic beta cell function under the pathological condition of diabetes mellitus through targeting Foxo1 and Sox6. Biochemical and biophysical research communications. 2019;519(2):294-301.

13. Song Y, Jin D, Jiang X, Lv C, Zhu H. Overexpression of microRNA-26a protects against deficient beta-cell function via targeting phosphatase with tensin homology in mouse models of type 2 diabetes. Biochemical and biophysical research communications. 2018;495(1):1312-6.

14. Yu CY, Yang CY, Rui ZL. MicroRNA-125b-5p improves pancreatic beta-cell function through inhibiting JNK signaling pathway by targeting DACT1 in mice with type 2 diabetes mellitus. Life sciences. 2019;224:67-75.

15. Liu YM, Ma JH, Zeng QL, Lv J, Xie XH, Pan YJ, et al. MiR-19a Affects Hepatocyte Autophagy via Regulating lncRNA NBR2 and AMPK/PPARalpha in D-GalN/Lipopolysaccharide-Stimulated Hepatocytes. Journal of cellular biochemistry. 2018;119(1):358-65.

16. Li Y, Luo T, Wang L, Wu J, Guo S. MicroRNA-19a-3p enhances the proliferation and insulin secretion, while it inhibits the apoptosis of pancreatic beta cells via the inhibition of SOCS3. International journal of molecular medicine. 2016;38(5):1515-24.

17. Alberti KG, Zimmet PZ. Definition, diagnosis and classification of diabetes mellitus and its complications. Part 1: diagnosis and classification of diabetes mellitus provisional report of a WHO consultation. Diabetic medicine : a journal of the British Diabetic Association. 1998;15(7):539-53.

18. Wang J, Lin Z, Yang Z, Liu X. lncRNA Eif4g2 improves palmitate-induced dysfunction of mouse beta-cells via modulation of Nrf2 activation. Experimental cell research. 2020;396(2):112291.

19. Ruan Y, Lin N, Ma Q, Chen R, Zhang Z, Wen W, et al. Circulating LncRNAs Analysis in Patients with Type 2 Diabetes Reveals Novel Genes Influencing Glucose Metabolism and Islet beta-Cell Function. 
Cellular physiology and biochemistry : international journal of experimental cellular physiology, biochemistry, and pharmacology. 2018;46(1):335-50.

20. Gao YP, Li Y, Li HJ, Zhao B. LncRNA NBR2 inhibits EMT progression by regulating Notch1 pathway in NSCLC. European review for medical and pharmacological sciences. 2019;23(18):7950-8.

21. Yu H, Xie Y, Zhou Z, Wu Z, Dai X, Xu B. Curcumin Regulates the Progression of Colorectal Cancer via LncRNA NBR2/AMPK Pathway. Technology in cancer research \& treatment. 2019;18:1533033819870781.

22. Wu X, Yu T, Ji N, Huang Y, Gao L, Shi W, et al. IL6R inhibits viability and apoptosis of pancreatic beta-cells in type 2 diabetes mellitus via regulation by miR-22 of the JAK/STAT signaling pathway. Diabetes, metabolic syndrome and obesity : targets and therapy. 2019;12:1645-57.

23. Wang S, Wen X, Han XR, Wang YJ, Shen M, Fan SH, et al. MicroRNA-30d preserves pancreatic islet beta-cell function through negative regulation of the JNK signaling pathway via SOCS3 in mice with streptozotocin-induced diabetes mellitus. Journal of cellular physiology. 2018;233(9):7343-55.

24. Feng S, Zhu X, Fan B, Xie D, Li T, Zhang X. miR19a3p targets PMEPA1 and induces prostate cancer cell proliferation, migration and invasion. Molecular medicine reports. 2016;13(5):4030-8.

25. Zhang B, Liu Y, Zhang J. Silencing of miR-19a-3p enhances osteosarcoma cells chemosensitivity by elevating the expression of tumor suppressor PTEN. Oncology letters. 2019;17(1):414-21.

26. Luo H, Xu C, Le W, Ge B, Wang T. IncRNA CASC11 promotes cancer cell proliferation in bladder cancer through miRNA-150. Journal of cellular biochemistry. 2019;120(8):13487-93.

27. Li FP, Lin DQ, Gao LY. LncRNA TUG1 promotes proliferation of vascular smooth muscle cell and atherosclerosis through regulating miRNA-21/PTEN axis. European review for medical and pharmacological sciences. 2018;22(21):7439-47.

28. Song M, Zou L, Peng L, Liu S, Wu B, Yi Z, et al. LncRNA NONRATT021972 siRNA normalized the dysfunction of hepatic glucokinase through AKT signaling in T2DM rats. Endocrine research. 2017;42(3):180-90.

29. Xu L, Li Y, Yin L, Qi Y, Sun H, Sun P, et al. miR-125a-5p ameliorates hepatic glycolipid metabolism disorder in type 2 diabetes mellitus through targeting of STAT3. Theranostics. 2018;8(20):5593-609.

30. Isin M, Uysaler E, Ozgur E, Koseoglu H, Sanli O, Yucel OB, et al. Exosomal IncRNA-p21 levels may help to distinguish prostate cancer from benign disease. Frontiers in genetics. 2015;6:168.

31. Alizadeh M, Safarzadeh A, Beyranvand F, Ahmadpour F, Hajiasgharzadeh K, Baghbanzadeh A, et al. The potential role of miR-29 in health and cancer diagnosis, prognosis, and therapy. Journal of cellular physiology. 2019;234(11):19280-97.

32. Li X, Zhao Z, Gao C, Rao L, Hao P, Jian D, et al. The Diagnostic Value of Whole Blood lncRNA ENST00000550337.1 for Pre-Diabetes and Type 2 Diabetes Mellitus. Experimental and clinical 
endocrinology \& diabetes : official journal, German Society of Endocrinology [and] German Diabetes Association. 2017;125(6):377-83.

33. Dehghani M, Aghaei Zarch SM, Vahidi Mehrjardi MY, Nazari M, Babakhanzadeh E, Ghadimi H, et al. Evaluation of miR-181b and miR-126-5p expression levels in T2DM patients compared to healthy individuals: Relationship with NF-kappaB gene expression. Endocrinologia, diabetes y nutricion. 2020;67(7):454-60.

34. Ghafouri-Fard S, Esmaeili M, Shoorei H, Taheri M. A comprehensive review of the role of long non-coding RNAs in organs with an endocrine function. Biomedicine \& pharmacotherapy $=$ Biomedecine \& pharmacotherapie. 2020;125:110027.

35. Qiao S, Xu Q, Cheng L, Zhang Y, Liu X, Zheng C, et al. Integrated analysis of differentially expressed lncRNAs in Medial Temporal Lobe Epilepsy. Neuro endocrinology letters. 2018;39(2):11924.

36. Cheng J, Yang A, Cheng S, Feng L, Wu X, Lu X, et al. Circulating miR-19a-3p and miR-483-5p as Novel Diagnostic Biomarkers for the Early Diagnosis of Gastric Cancer. Medical science monitor : international medical journal of experimental and clinical research. 2020;26:e923444.

37. Bai R, Cui Z, Ma Y, Wu Y, Wang N, Huang L, et al. The NF-kappaB-modulated miR-19a-3p enhances malignancy of human ovarian cancer cells through inhibition of IGFBP-3 expression. Molecular carcinogenesis. 2019;58(12):2254-65. 


\section{Figure legends}

Figure 1. Effects of NBR2 on pancreatic $\beta$ cell insulin secretion and proliferation. A. The relative expression level of NBR2 was increased by pcDNA3.1-NBR2, while was decreased by NBR2 siRNA.

B. Glucose-stimulated insulin secretion was inhibited by NBR2 overexpression, while was enhanced by NBR2 knockdown in INS-1 cells. C. The proliferation was inhibited by the NBR2 overexpression, and was promoted by the NBR2 knockdown. $(* P<0.05, * * P<0.01, * * * P<0.001)$

Figure 2. MiR-19a-3p directly mediated the regulatory effects of NBR2 on pancreatic $\beta$ cell function. A. A complementary binding site of NBR2 and miR-19a-3p. B. The miR-19a-3p expression was promoted by the miR-19a-3p mimic compared with that in untransfected cells. C. Relative luciferase activity was detected in INS-1 cells co-transfected with NBR2-WT or NBR2-MUT and miR-19a-3p NC or miR-19a $-3 p$ mimic by dual-luciferase reporter assay. D. NBR2 overexpression suppressed and NBR2 knockdown enhanced the expression of miR-19a-3p in INS-1 cells. E. miR-19a-3p overexpression reversed the inhibitory effect of NBR2 overexpression on the miR-19a-3p expression in INS-1 cells. F. The insulin secretion inhibited by NBR2 overexpression was reversed by miR-19a-3p overexpression. G. The cell proliferation inhibited by NBR2 overexpression was reversed by miR-19a-3p overexpression. $(* P<0.05$, ${ }^{* *} P<0.01,{ }^{* * *} P<0.001$ vs. Untransfected; ${ }^{\#} P<0.05,{ }^{\# \#} P<0.001$ vs. pcDNA3.1-NBR)

Figure 3. Relative expression of the NBR2/miR-19a-3p axis in T2DM patients. A. The expression level of serum NBR2 in T2DM patients and healthy volunteers. B. Serum miR-19-5p expression in T2DM patients and healthy volunteers. C. A negative correlation was found between serum miR-19a-3p level and serum NBR2 level $(\mathrm{r}=-0.858, P<0.001) .(* * * P<0.001)$

Figure 4. Correlation of the NBR2/miR-19a-3p axis with FPG in T2DM patients $(r=0.725, P<0.001)$. A. A positive correlation was found between serum NBR2 level and FPG. B. A negative correlation was found between serum miR-19a-3p level and FPG $(\mathrm{r}=-0.685, P<0.001)$.

Figure 5. Diagnostic performance of the NBR2/miR-19a-3p axis in T2DM patients. A. A ROC curve of NBR2. B. A ROC curve of miR-19a-3p. C. A ROC curve of NBR2 + miR-19a-3p. AUC, area under the curve. 
Table 1 Baseline characteristics of the study subjects

\begin{tabular}{cccc}
\hline Variables & $\begin{array}{c}\text { Healthy controls } \\
(\mathrm{n}=62)\end{array}$ & $\begin{array}{c}\text { T2DM patients } \\
(\mathrm{n}=106)\end{array}$ & $P$ value \\
\hline Gender & & & \\
Female & 26 & 47 & 0.762 \\
Male & 36 & 59 & 0.526 \\
Age $($ years $)$ & $47.129 \pm 13.255$ & $48.660 \pm 16.031$ & 0.395 \\
BMI $\left(\mathrm{kg} / \mathrm{m}^{2}\right)$ & $24.069 \pm 3.976$ & $24.844 \pm 6.476$ & 0.384 \\
TC $(\mathrm{mM})$ & $4.581 \pm 0.722$ & $2.504 \pm 1.352$ & 0.238 \\
TG $(\mathrm{mM})$ & $2.279 \pm 0.830$ & $1.192 \pm 0.293$ & 0.123 \\
HDL $(\mathrm{mM})$ & $1.264 \pm 0.286$ & $2.641 \pm 0.990$ & 0.084 \\
LDL $(\mathrm{mM})$ & $2.353 \pm 1.110$ & $9.138 \pm 2.520$ & $<0.001$ \\
HbA1c $(\%)$ & $5.564 \pm 0.796$ & $9.189 \pm 2.141$ & $<0.001$ \\
OGTT 0h (mM) & $4.856 \pm 0.455$ & $15.251 \pm 3.558$ & $<0.001$ \\
\hline OGTT $2 \mathrm{~h}(\mathrm{mM})$ & $5.825 \pm 1.537$ & & \\
\hline
\end{tabular}

BMI, body mass index; TC, total cholesterol; TG, triglyceride; HDL, high density lipoproteins; LDL, low density lipoproteins; HbA1c, glycosylated hemoglobin; OGTT, oral glucose tolerance test. 
Table 2. Primer sequences used for qRT-PCR

\begin{tabular}{ccc}
\hline Gene & \multicolumn{2}{c}{ Primer sequences } \\
\hline NBR2 & Forward & 5'-GGAGGTCTCCAGTTTCGGTA-3' \\
& Reverse & 5'-TTGATGTGTGCTTCCTGGG-3' \\
miR-19a-3p & Forward & 5'-GCCGAGTGTGCAAATCTAT-3' \\
& Reverse & 5'-CTCAACTGGTGTCGTGGA-3' \\
GAPDH & Forward & 5'-CCATGGGGAAGGTGAAGGTC-3' \\
& Reverse & 5'-GAAGGGGTCATTGATGGCAAC-3' \\
U6 & Forward & 5'-CTCGCTTCGGCAGCACA-3' \\
& Reverse. & 5'-CTCGCTTCGGCAGCACA-3' \\
\hline
\end{tabular}



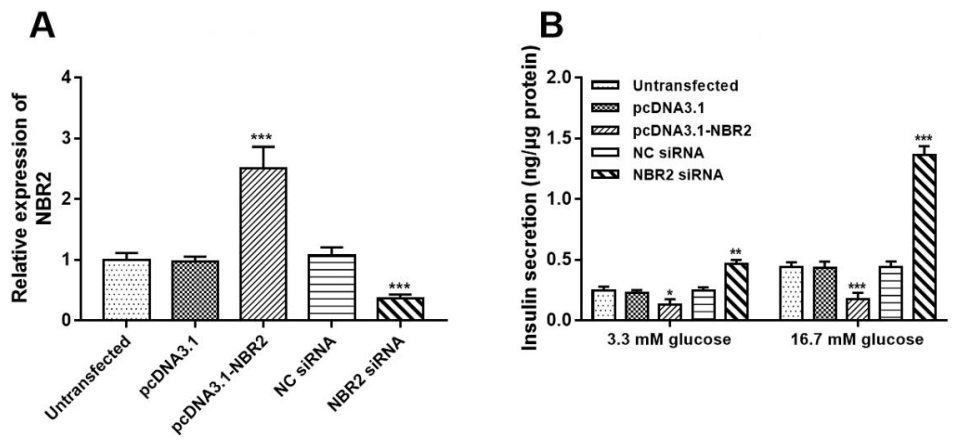

\section{C}

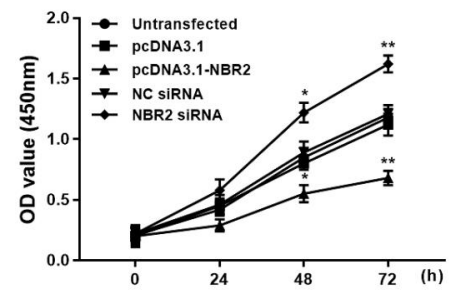

Effects of NBR2 on pancreatic $\beta$ cell insulin secretion and proliferation. 

A
NBR2-WT 5'-CUUGUUCUUUUUUAUGAUUGCACA-3'
miR-19a-3p 3'-AGUCAAAACGUAUCUAAACGUGU-5'
NBR2-MUT 5'-CUUGUUCUUUUUUAUGAGCUGUAA-3'

B

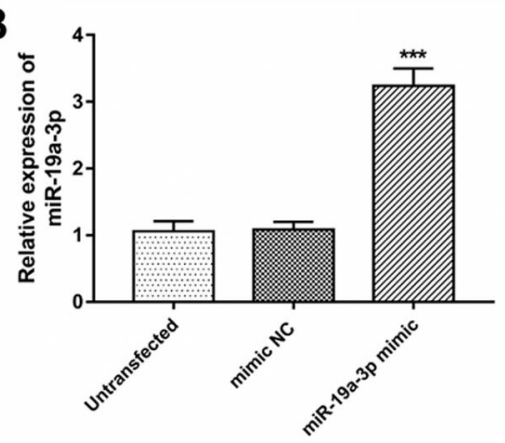

D

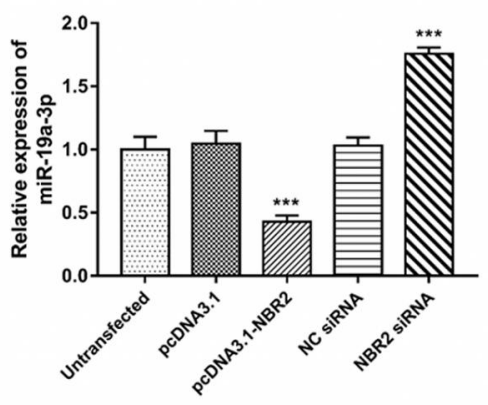

F

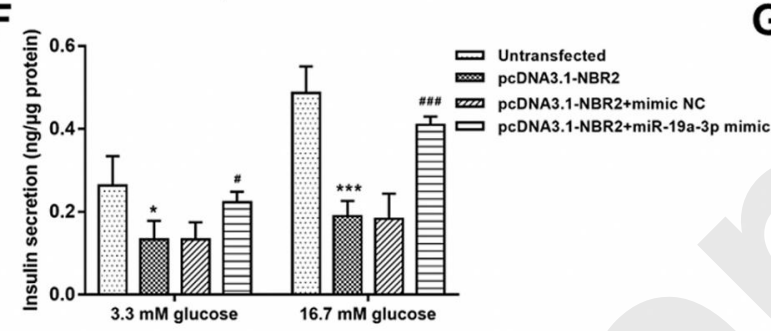

C

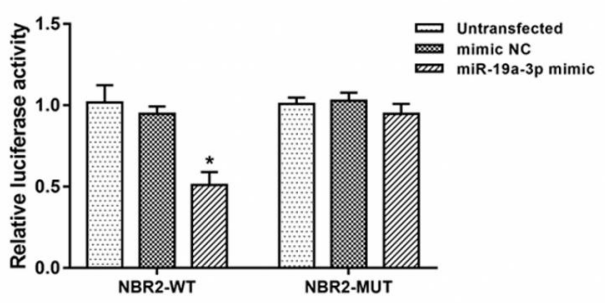

E
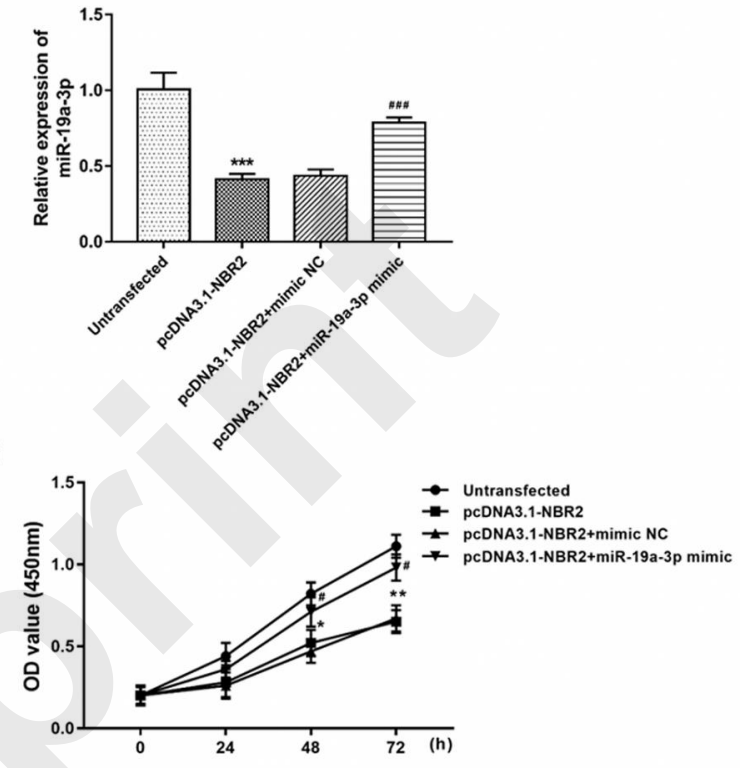

MiR-19a-3p directly mediated the regulatory effects of NBR2 on pancreatic $\beta$ cell function. 

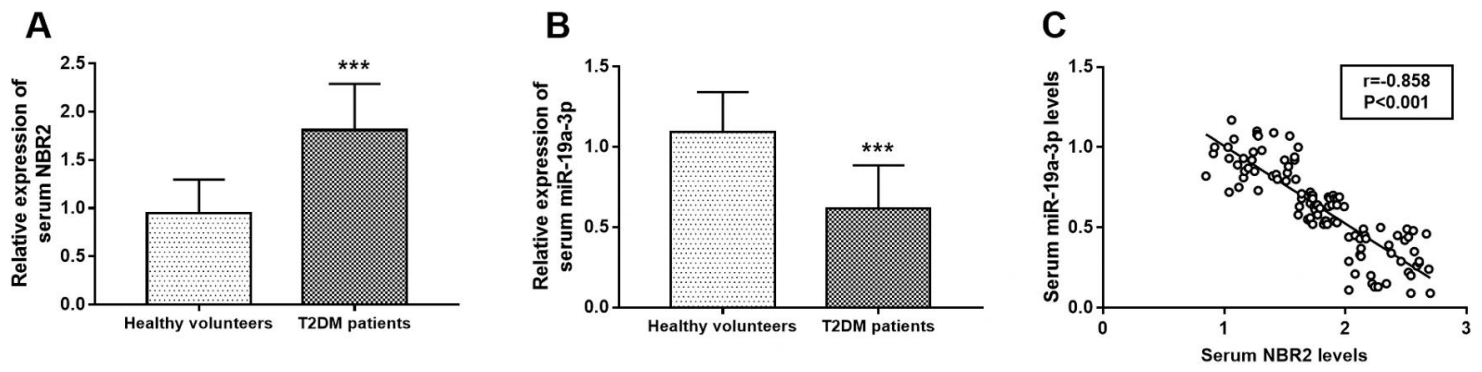

Relative expression of the NBR2/miR-19a-3p axis in T2DM patients. 
A

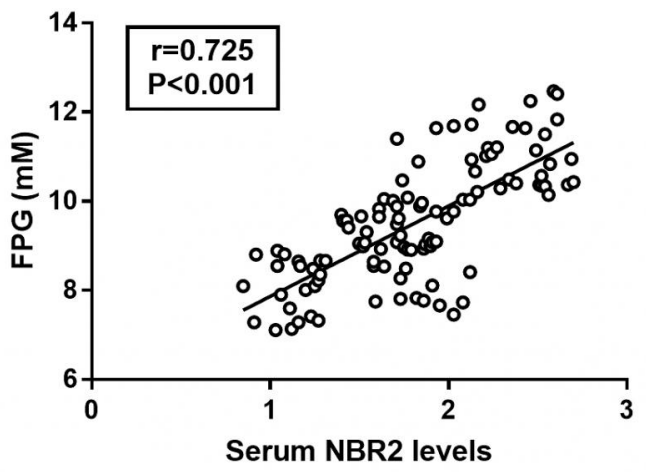

B

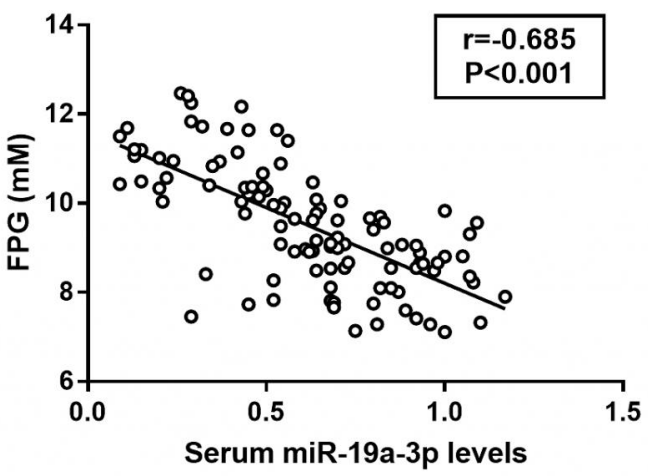

Correlation of the NBR2/miR-19a-3p axis with FPG in T2DM patients ( $r=0.725, P \square 0.001)$. 

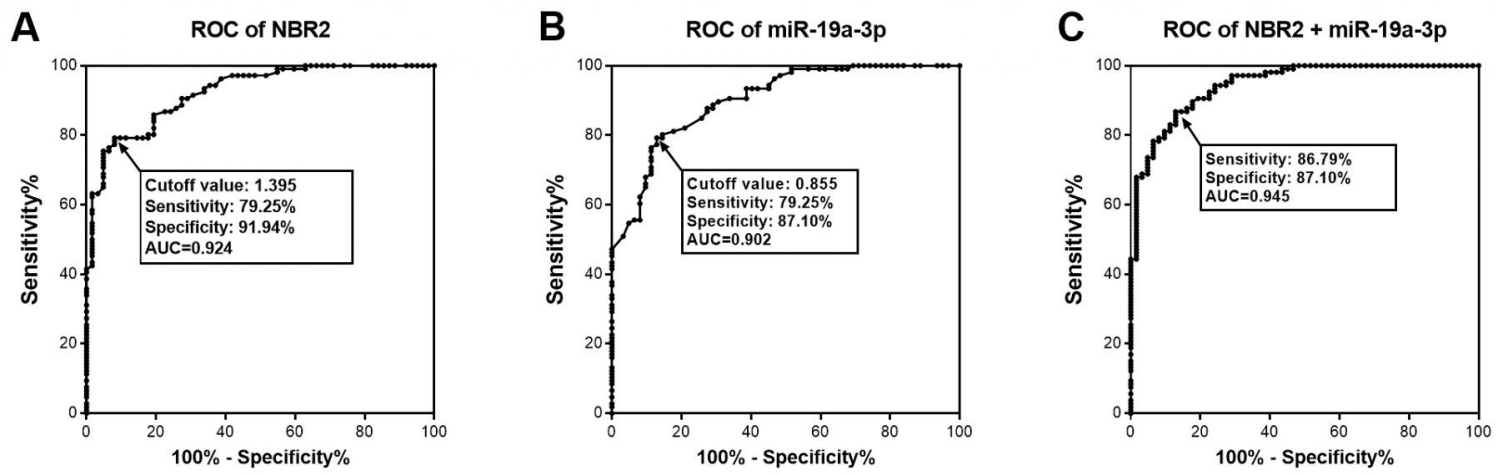

Diagnostic performance of the NBR2/miR-19a-3p axis in T2DM patients. 